\title{
Numerical Simulation of the Polymer Electrolyte Membrane Electrolyzer
}

\author{
Alexandre Tugirumubano ${ }^{1}$, Hee Jae Shin ${ }^{2}$, Lee Ku Kwac ${ }^{3}$, Hong Gun Kim ${ }^{4}$ \\ ${ }^{1}$ (Department of Mechanical Engineering/ Jeonju University, South Korea) \\ ${ }^{2}$ (Jeonju University Institute of Carbon Technology/ Jeonju University, South Korea) \\ ${ }_{3}^{3}$ (Department of Manufacturing Technology and Design Engineering/Jeonju University, South Korea) \\ ${ }^{4}$ (Department of Mechanical and Automotive Engineering/ Jeonju University, South Korea)
}

\begin{abstract}
In the development of renewable energy, polymer electrolyte membrane(PEM) electrolyzer has shown an impressive impact on the production of pure hydrogen gas, that can be used as fuel and energy storage, and oxygen gas. In this study, the numerical simulation was conducted in order to investigate I-V characteristic of PEM electrolyzer. Moreover, the electromechanical performance that is characterized by the polarization curve depends on many parameters. Therefore, the impact of the membrane and electrodes thicknesses and electrodes porosities are presented.
\end{abstract}

Keywords: mass transport, charge transport, performance, electrolyzer

\section{Introduction}

Recently, the use of hydrogen gas presents many applications, such as in fuel cell, electrical car, energy storage etc, so that its demand increases with time. Therefore, it is required a good quality, and mass production of hydrogen gas. Among different ways of hydrogen production that exist the polymer electrolyte membrane water electrolysis is the most attractive and promising technology due to its high efficiency, high purity of gas production, and friendlier to the environment [1-2]. Not only hydrogen is produced but also oxygen gas is obtained by this technology.

The PEM water electrolysis, that uses the solid polymer as electrolyte, decomposes water that is supplied to the anode into oxygen, protons and electrons at the interface of the membrane and the anode electrode by the effect of electric energy [3-4]. Then the oxygen is removed and electrons travel through the electric conductor while the protons cross through the membrane to the interface of cathode electrode and membrane where they combine with the electrons to form the hydrogen gas by oxidation reaction. The produced hydrogen can be used directly or be stored in the containers for later application.

Although this technology produces good quality of gas with less emission, it is very expensive due to the high cost of its components. In our previous study, we have established the effect of different operating parameters and constants on the performance of the PEM electrolyzer, in which we found that the increase of the temperature, charge transfer coefficient exchange, and current density of the electrodes can increase the performance whereas the increase of the operating pressure reduces it[5].In this work, we conducted a numerical analysis for charge and mass transport where the impact of design parameters of membrane electrode assembly(MEA), which is the assembly of membrane, catalysts and the gas diffusion layers, on the PEM electrochemical behavior are investigate.

\section{Modeling}

The PEM electrolyzer model was designed in COMSOL Multiphysics (see Fig.1). The considered geometry consists of cathode catalyst (made of Platinum and carbon (Pt/C)), anode catalyst(Iridium), cathode gas diffusion layer (Carbon Paper), anode gas diffusion layer(Titanium), and the membrane), serpentine bipolar plates made of Titanium. The Secondary Current Distribution and the reacting flow in porous media were set up in order to solve the electronic and mass transfer phenomena. The Bulter-Volmer models were used for the electrochemistry of the electrolyzer, while the species flow is modelled by the Navier-Stokes equations. The conductivity of the membrane, which depends on the temperature and the membrane humidity, was used as variable and defined according to ref. [2,6]. In PEM electrolyzer, there is always the mixture of oxygen and water in the anode compartment and sometimes a mixture of a very small amount of water and hydrogen gas in the cathode compartment.

The transport of these fluids is a diffusion in parous medias and free flow in plates' channels. Therefore, we defined the effective binary diffusion coefficients of mixtures as in ref. [5-6]. In this analysis, the operating temperature was $40^{\circ} \mathrm{C}$, the operating pressure was $1 \mathrm{~atm}$, the porosity of 0.3 was used for both catalysts while the exchange current densities of cathode and anode were $0.027 \mathrm{~A} / \mathrm{cm}^{2}$ and $5 \times 10^{-6} \mathrm{~A} / \mathrm{cm}^{2}$ respectively. The voltage values of $1.4 \mathrm{~V}$ to $2.4 \mathrm{~V}$ were applied on the anode bipolar plates and the water molar fraction 0.95 
was set at the anode inlet.The analysis was performed with a stationary study and direct PARDISO method was used as solver due to its rapidity compared to another solver that exist in COMSOL.

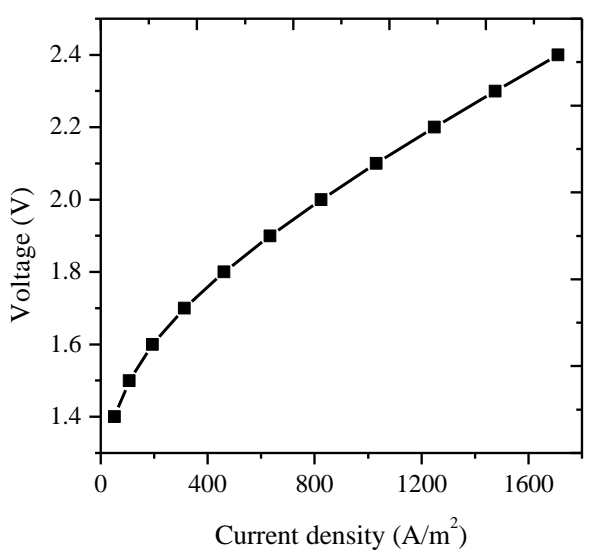

Figure 1. Geometry model of PEM electrolyzer

\subsection{Electrochemical performance}

\section{Results and discussion}

The performance of an PEM electrolyzer is characterized by the I-V curve as shown in Fig.2. It can be seen form the polarization curve that when the applied voltage increases, the current density also increase.

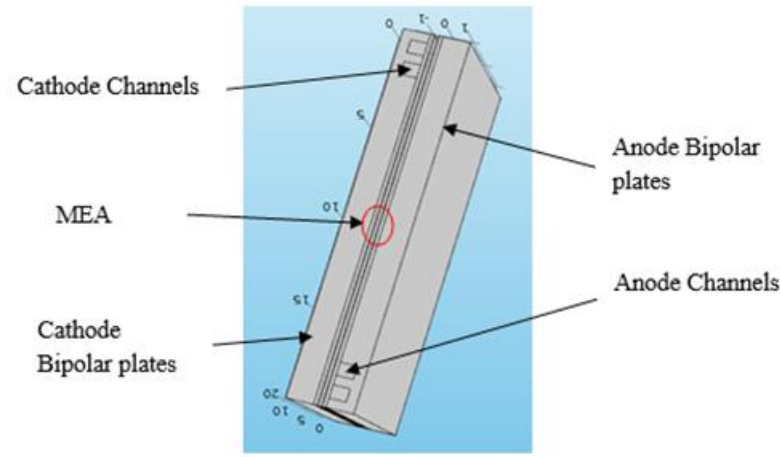

Figure 2. The polarization curve of PEM electrolyzer

\subsection{Effect of membrane and electrodes' thickness}

The membrane thickness was defined as parameters of $0.16 \mathrm{~mm}$ to $0.22 \mathrm{~mm}$ in order to investigate their impact on the electrolyzer performance. The results shown in Fig.3 indicated that the thicker membrane could lead to low performance. In fact, when the membrane thickness is large, the electric and ionic resistance increase which causes the voltage loss, and therefore high ohmic loss. However, this effect is less obvious at low cell voltages. The same was observe for the thickness of the electrode where it was varied form $0.25 \mathrm{~mm}$ to $0.15 \mathrm{~mm}$ for cathode gas diffusion layer and from $0.2 \mathrm{~mm}$ to $0.1 \mathrm{~mm}$ for the anode gas diffusion layer as shown in Fig.4 and Fig.5.

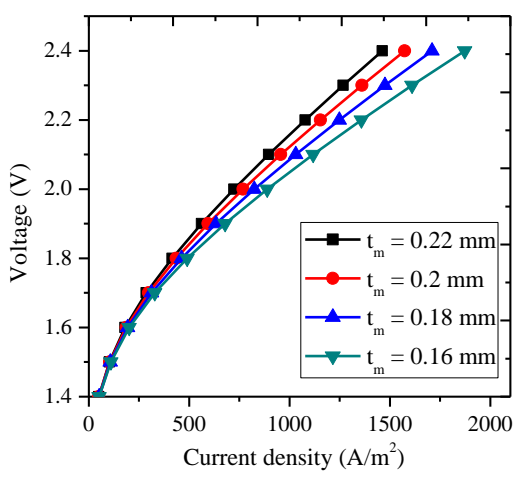

Figure 3. Effect of membrane thickness on the performance 


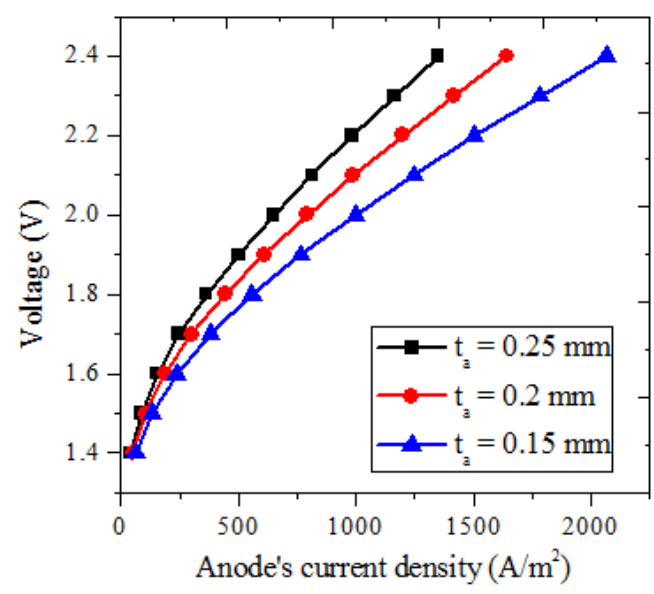

Figure 4. Effect of anode GDL's thickness on the performance

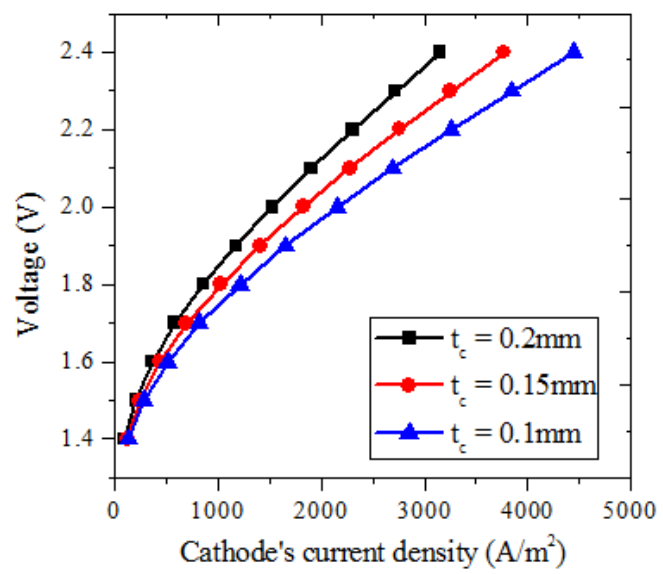

Figure 5. Effect of cathode GDL's thickness on the performance

\subsection{The effect of the electrodes' porosity}

The porosity is very important design parameter for the diffusion of the species through the components of an electrolytic cell. In PEM electrolyzer, the diffusion usually occurs across the gas diffusion layers and the catalysts supplying the water and discharging the products from the interface of the membrane and the electrodes. Therefore, different values of porosity were given to the gas diffusion layers where they were $0.4,0.6$, and 0.8 for the anode gas diffusion layer, and $0.3,0.5$ and 0.7 for the cathode gas diffusion layer. The parametric study was conducted by varying the porosity of one electrode while the other's porosity was kept constant. The results are plotted in Fig.6 and Fig.7. The results showed that the electrode with high porosity lead to a better performance of the PEM electrolyzer at high cell voltages. In fact, the electrode with high porosity have a low volume fraction, and therefore less electric resistance. In addition, the increment of the porosity reduces the diffusion voltage loss and improve the cell's performance.

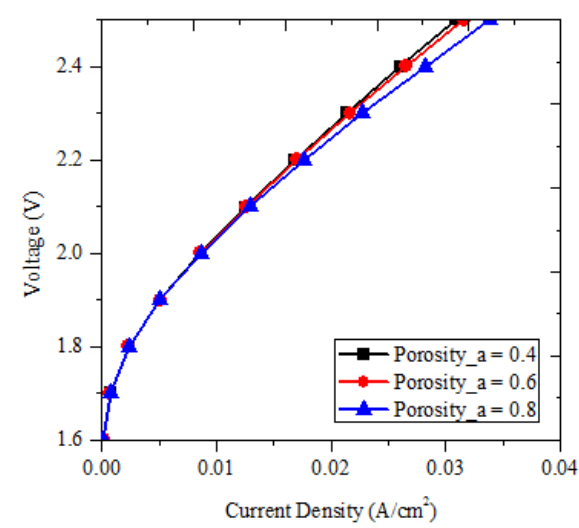

Figure 6. Effect of anode electrode porosity on the performance 


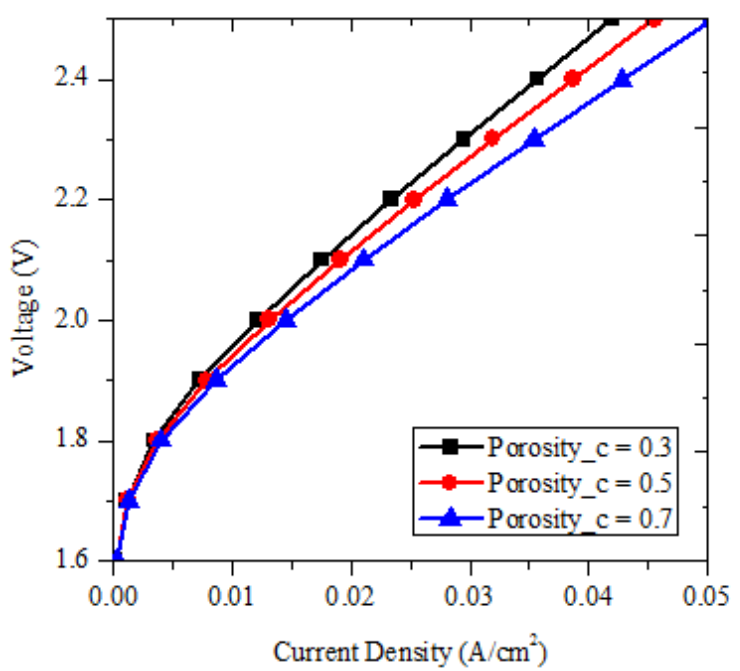

Figure7. Effect of cathode electrode porosity on the performance

\section{Conclusions}

A model of a polymer electrolyte membrane electrolyzer with serpentine flow field channels was designed and the simulation was conducted using COMSOL Multiphysics. The polarization curve was plotted and the impact of some geometric design parameters of the membrane electrolyte assembly(MEA) on the cell's electrochemical behavior was investigated. It was found that the thinner the membrane or electrodes are the least cell voltages are required for high current density and therefore the better performance of the electrolyzer. The porosity of electrodes showed a significant impact on the manufacturing of PEM electrolyzer where the electrodes with high porosity are preferable for less voltage losses and good performance. The result from this work can be used in decision making during the production the electrolyzer's components.

\section{Acknowledgements}

This research was supported by basic science research program through the National Research Foundation of Korea(NRF) funded by the ministry of education, Science and technology (No.2013R1A1A2061581 and financially supported by the National Foundation of Korea(NRF) funded by the Korea Government(MISP) (No.2014R1A2A1A11054594) and (No.2014R1A2A1A11053533).

\section{References}

[1] P. Choi,D.G. Bessarabov, R.Datta, A simple model for solid polymer electrolyte(SPE) water electrolysis, Solid State Ionics 175, 2004, 535-539.

[2] B. Han, M. S. Stuart, J. Mo, F. Y. Zhang, Electrochemical performance modeling of a proton exchange membrane electrolyzer cell for hydrogen energy, International Journal of Hydrogen Energy 40, 2015, 7006-7016.

[3] M. Ni, M.K.H.Leung, D.Y.C.Leung, Electrochemistry modeling of proton exchange membrane(PEM) water electrolysis for hydrogen production, World Hydrogen Energy Conference 16,2006, 13-16.

[4] P. Millet, N. Mbemba, S.A. Grigoriev, V.N. Fateev A. Aukauloo. C. Etievant, Electrochemical performances of PEM Water electrolysis cells and perspectives, International Journal of Hydorgen Energy 36(6), 2011, 4134-4142.

[5] T.Alexndre, Analysis of polymer electrolye membrane(PEM) electrolyzer performance using 3-D mesh, Master of Science Thesis, Jeonju University, Jeonju, 2016.

[6] F.Marangio, M.Santarelli, M.Cali, Theoritical model and experimental analysis of a high pressure PEM water electrolyser for hydrogen production, International Journal of Hydrogen Energy 34, 2009, 1143-1158. 